\title{
Effective strategy for improving the dielectric strength and insulation lifetime of LLDPE
}

Xiaotian Li, ${ }^{\dagger,}$ Qiyang Guo, ${ }^{*} \dagger$ Xiaopeng Sun, ${ }^{\dagger}$ Fanghong Yang, ${ }^{\dagger}$ Wenfei Li, ${ }^{\dagger}, \star$ Zhanhai

$$
\mathrm{Yao}^{*, \dagger}
$$

$\dagger^{\dagger}$ Laboratory of Polymer Physics and Chemistry, Changchun Institute of Applied Chemistry, Chinese Academy of Sciences, Changchun130022, People's Republic of China

* Department of Chemistry and Environmental Engineering, Changchun University of Science and Technology, Changchun 130022, People's Republic of China State Key

Correspondence to: Q. Guo (qyguo1991@,ciac.ac.cn), Z. Yao (yaozh@,ciac.ac.cn)

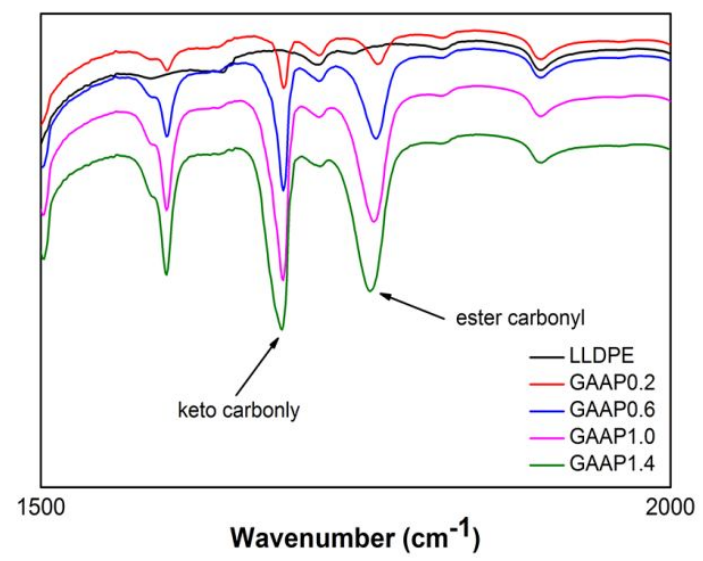

Figure S1. Calibration FTIR spectra of LLDPE-g-AAP with different AAP concentration. 


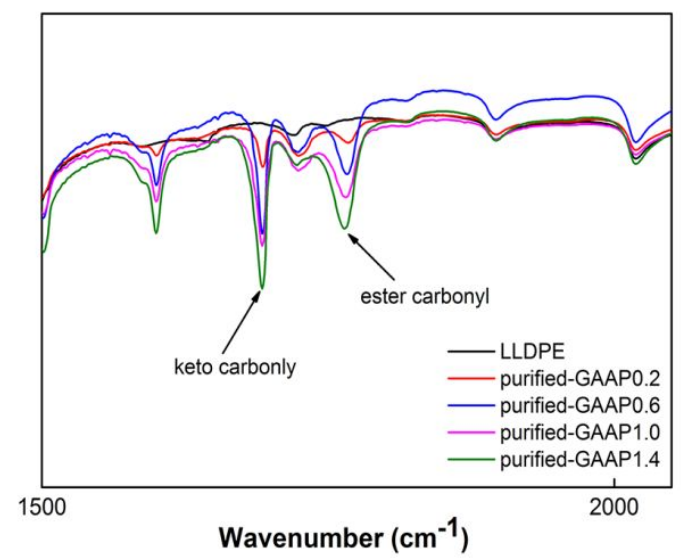

Figure S2. The FTIR spectra of ester and keto carbonyl in purified LLDPE-g-AAP samples with different initial AAP concentration.

Table S1. The results of Gr and GE for LLDPE-g-AAP with different content of AAP

$\begin{array}{ccc}\text { Sample } & \text { Gr }(w t \%) & \text { GE }(\%) \\ \text { GAAP0.2 } & \mathbf{0 . 0 7} & \mathbf{3 5} \\ \text { GAAP0.6 } & \mathbf{0 . 2 7} & \mathbf{4 5} \\ \text { GAAP1.0 } & \mathbf{0 . 5 0} & \mathbf{5 0} \\ \text { GAAP1.4 } & \mathbf{0 . 5 6} & \mathbf{4 0}\end{array}$



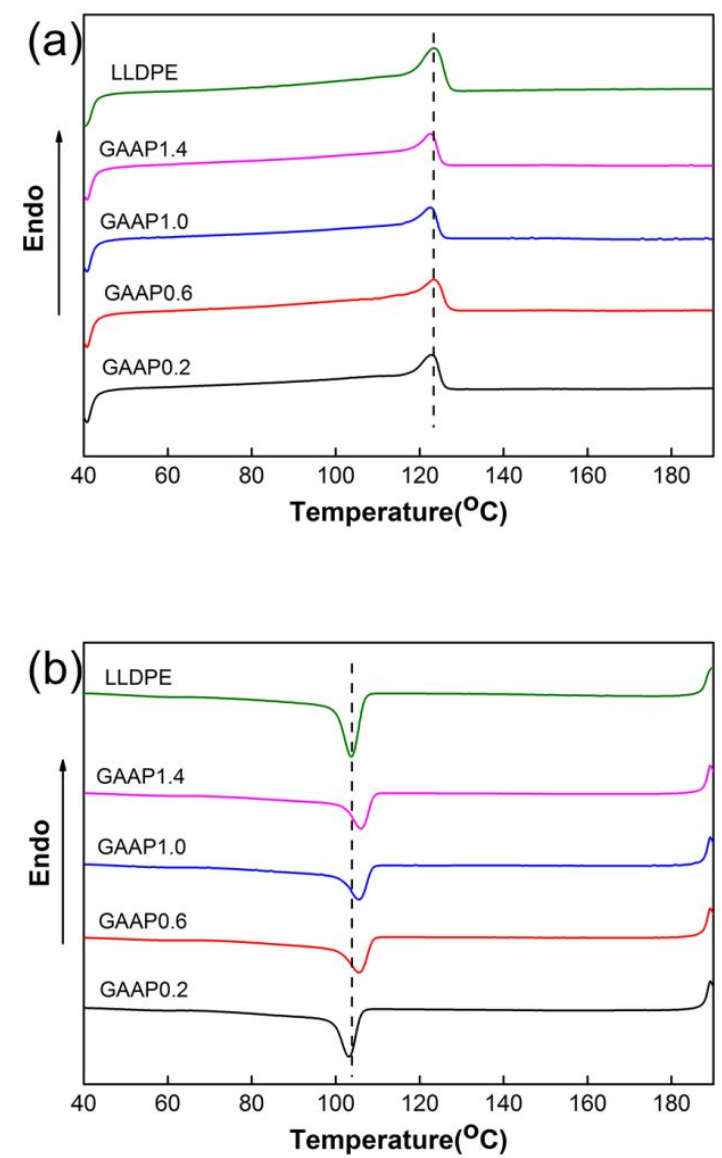

Figure S3. (a) DSC melting curves and (b) crystallization curves of LLDPE and LLDPE-g-AAP graft copolymers. 

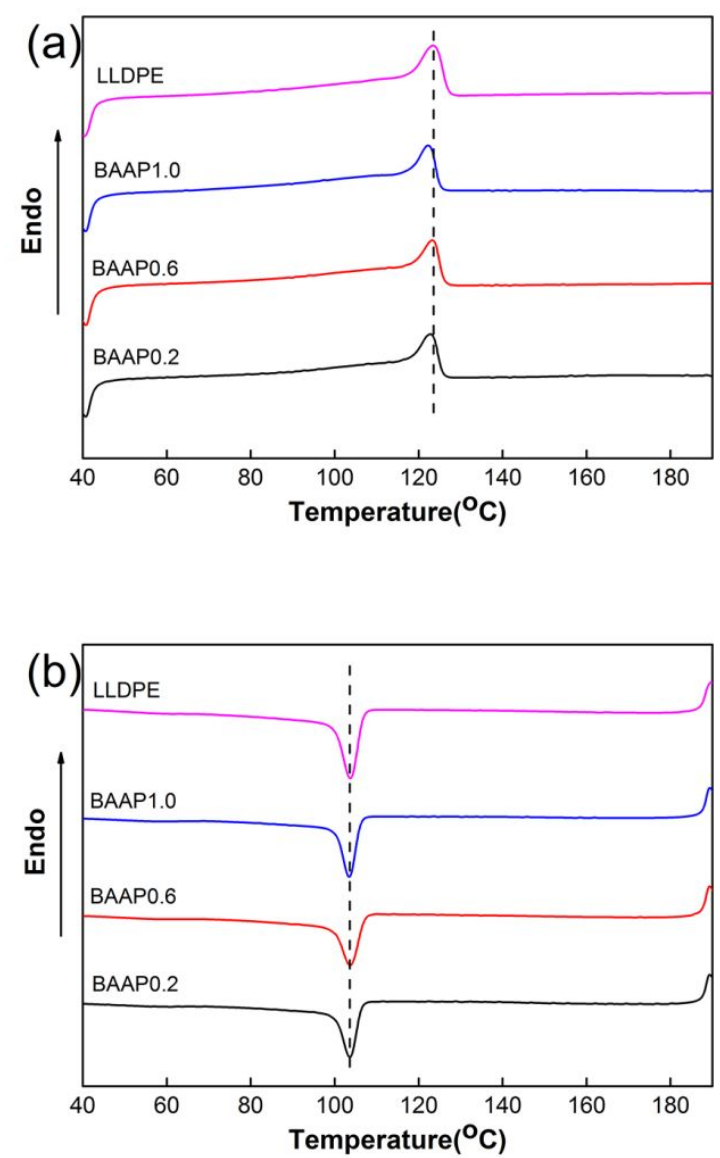

Figure S4. (a) DSC melting curves and (b) crystallization curves of LLDPE and LLDPE/AAP blends.

Table S2. The DC breakdown strength of LLDPE-g-AAP treated at $60{ }^{\circ} \mathrm{C}$ for different times.

\begin{tabular}{cccccc} 
Sample & \multicolumn{5}{c}{$\alpha(\mathrm{kV} / \mathrm{mm})$} \\
& $24 \mathrm{~h}$ & $48 \mathrm{~h}$ & $96 \mathrm{~h}$ & $192 \mathrm{~h}$ & $288 \mathrm{~h}$ \\
LLDPE & $\mathbf{2 6 9 . 3}$ & $\mathbf{2 7 0 . 8}$ & $\mathbf{2 7 4 . 5}$ & $\mathbf{2 7 8 . 1}$ & $\mathbf{2 7 6 . 6}$ \\
GAAP0.2 & $\mathbf{4 1 6 . 9}$ & $\mathbf{4 0 2 . 9}$ & $\mathbf{3 9 3 . 2}$ & $\mathbf{3 8 9 . 6}$ & $\mathbf{3 8 2 . 1}$ \\
GAAP0.6 & $\mathbf{4 0 6 . 5}$ & $\mathbf{4 0 2 . 0}$ & $\mathbf{4 0 9 . 4}$ & $\mathbf{4 0 6 . 7}$ & $\mathbf{4 1 0 . 5}$ \\
GAAP1.0 & $\mathbf{4 1 4 . 0}$ & $\mathbf{4 0 8 . 6}$ & $\mathbf{4 1 5 . 8}$ & $\mathbf{4 2 1 . 0}$ & $\mathbf{4 2 3 . 0}$ \\
GAAP1.4 & $\mathbf{4 0 4 . 8}$ & $\mathbf{4 1 2 . 8}$ & $\mathbf{4 1 5 . 1}$ & $\mathbf{4 2 0 . 5}$ & $\mathbf{4 3 0 . 2}$
\end{tabular}

